\title{
IMPLEMENTING EVIDENCE-BASED STRATEGIC DECISION MAKING IN HER INSTITUTIONS: THE CASE OF PARIS SCIENCES ET LETTRES UNIVERSITY ${ }^{1}$
}

\author{
EMMANUEL BASSET AND MATTHIAS HEUSER \\ DOI: $10.22163 /$ fteval.2019.341
}

\section{ABSTRACT}

$\mathrm{T}$

he design and implementation of impact-oriented R\&l policies depends on the capacity of contextualizing the expected impact of such policies. However, most of the available data sources for R\&l policies are still fragmented, highly heterogeneous and not interoperable. Public policy and strategic decision making suffer from lack of integration of existing data, which are available from separate sources, follow different definitions, have disparate time scales. In such a scenario, decision makers and policy makers can still easily stumble on spurious correlations when indicators and relevant data are deprived of context and maintained in vertically separated containers or 'silos'. Silos of data remain separate, non-integrated, often not even interoperable.

In other terms, to inform Ryl policies and design impact-oriented approaches, data that are currently dispersed and highly heterogeneous, need to be accessed in an integrated, unified and semantically consistent way. To achieve this, a combination of semantic-based technological solutions and open government approach must be increased. This, however, does not imply that the problem of quality and pertinence of specific indicators for the uses retained is solved by this technological choice.

\section{INTRODUCTION}

This paper presents as a case study the project of establishing a comprehensive Research Information System (RIS) in University Paris Sciences \& Letters $^{2}$ (PSL), a key institution in the Parisian Higher Education, Research and Innovation (HERI) landscape. The system has been developed for integrating distributed and heterogeneous data sources for (1) informing top-level strategic decision-making at university level in the context of a period of radical change in the French HERI system as well as (2) open up the university to other quadruple helix actors by providing a detailed research portfolio and (3) generally increase the availability of pertinent data to mid-level management and individual researchers, fostering a culture change towards data use. It relies on the Semantic Web technological framework to extract meaningful insights from extensive and heterogeneous data and aims at a powerful contribution to the Open Science movement.

Our research question is therefore: how to tap the potential of current developments to overcome the difficulties attached to the implementation of a RIS at university-level and how to maximise its chances of success?

\section{CONTEXT}

Like many areas of public and private administration, HERI institutions are taken in the recent revolutions in the use of data for policy design and strategic decision-making. The "University 4.0", as we might mockingly call it, is suggested as the new model for rational and evidence-based development of research and higher education.

Measuring scientific production through several categories of quantitative indicators (e.g. number of publications, number of citations related to these publications, number of patents related to research, number of research grants obtained) has become common practice in all fields of academic life: students and staff may choose universities according to international rankings that rely heavily on quantitative indicators (ARWU, CWTS Leiden, THE, OS are the most famous examples); expert committees may look at values such as the h-index to hire or promote researchers; national agencies may allocate funds to universities or research programs proportionally to the number of publications accepted by journals with high impact factors ${ }^{3}$. Although the use of such indicators

The authors acknowledge the STI 2018 Leiden conference, from which this template was adapted.

www.psl.eu

The first forms of evidence-based policy-making in the context of HERI as it is relevant today is linked to the introduction of systematic performance assessment sometimes correlating directly performance to funding allocation, the UK Research Assessment Exercise (RAE), established in 1986, being the first national research funding system based on past research performance. The UK reform served as reference for some other countries who introduced elements of performance-based funding in the 2000s, generally run either directly by Ministries (Norway), dedicated agencies (Flanders in Belgium, Italy and Spain) or research councils (Australia). In 2010, at least fourteen performance-based research funding systems were found across Europe and other countries like Australia, New Zealand, Hong Kong and China. Further information in Hicks (2013). 
is still highly debated, they are used increasingly by stakeholders, from students to faculty and administrative staff, so that the impact of such indicators has somewhat short-circuited debates about their pertinence.

In this situation, Higher Education Institutes (HEI) will need to find a middle ground between a purely pragmatic stance, prioritising making the most of the situation by profiting from the levers for visibility the indicators might provide, and a critical one, prioritising the improvement of the system. But as said above, HERI institutions are not alone in these developments. At regional, national, and international levels, sophistication in the use of evidence for defining research and innovation strategy and implementation has been growing, as have demands for public accountability. This means that the dialog between policy makers and actors of the regional/national/supranational ecosystems becomes more and more sophisticated as well. We see only the beginning of this in France, where national evaluation and funding organisms still rely on somewhat crude information systems and demands are often ad hoc and fairly disorganised. Still, with an eye on the developments in Great Britain and the Research Excellence Framework ${ }^{4}$, it does seem reasonable for an HERI actor to prepare for future developments in the direction of organised use of data for policy making across different levels and actors.

Especially since the publication of the Shanghai ranking in 2003, the pressure on universities increased to adapt to new quantified standards of excellence which are different (and often in part contradictory) with established procedures of quality-insurance mostly based on peerreview. As Thoenig and Paradeise put it: "Excellence rankings induce significant consequences for the very definition of academic quality. Universities are split between two quality regimes: a traditional one based on reputation and expressed through cardinal judgements delivered by insiders, and a new one based on quantitative ordinal scales invented by rankings of excellence as defined by outsiders" ${ }^{\prime \prime}$. One should not underestimate the significance of this shift: does generalisation of use of externally constructed data undermine universities' "strategic capacity" (Thoenig and Paradeise 2016)?

This point has been a key motivation for PSL to build a performant research intelligence system. Instead of continuously finding oneself confronted with externally defined indicator systems, PSL wished to build up internal competence and internal data to reclaim if not sovereignty, at least a strong voice on the modalities of evaluation of its work. More broadly, PSL saw the implementation of a RIS as an opportunity to foster acceptance of data within the academic community, and to find the right balance between quantitative evidence with existing qualitative procedures of quality-assessment.

A main point of diagnosis that constitutes a second key motivation and that will explain quite some decisions made concerning the design of the system at PSL is that institutions should take care of their own data. Data must (in most cases) be curated at its source and there must be a proper feedback loop with its users, that are, in our situation also its main producers who are a key element in the production of quality data. Else, data is likely not to reflect the reality of the research activity it is supposed to represent. Everyone needs good data for their own purposes.

However, if data systems do not connect between them, aggregation and collaboration become difficult. This goes for the inner organisation of universities as well as for their relations to the exterior. Therefore, PSL chose a scalable system based on a technology mix apt to prepare it for interoperability with other systems. This allows the integration of data internally, but also, potentially, towards the outside (other HERI actors, our region, the national ministry, the EU, or the public). Moreover, the Linked (Open) Data (LOD) approach allows us to contextualise internal data with external sources. This way, we can weight e.g. internal information on publications against external bibliometric data, clinical trial data, national or EU projects or against patents. LOD enables multiple interoperable perspectives.

\section{THE PROJECT PARIS SCIENCES ET LETTRES}

Paris Sciences et Lettres University (PSL) is a research-intensive Parisian University system or sometimes coined "collegiate university" established in 2010. It is not a fully integrated university at this point but engaged in a densification process from which it shall emerge as "one" university in some years' time. This transformation has been engaged during the excellence initiative, which provided PSL with a 750M€ endowment on the condition that it becomes a single university. PSL comprises 9 full members, all small and highly selective grands écoles and a range of associates, many of which are strong institutions with their own history and independent reputation like the École Normale Supérieure de Paris, the Paris School of Mines or the Paris Observatory, the most important research centre on astronomy in France. As said, it is amid a profound institutional reconfiguration, which on the one hand explains the present initiative and on the other makes it difficult because of a complicated political and administrative situation.

\section{OBJECTIVES}

Penfield and al. (2014) identify four primary purposes of measuring research impact:

1. Monitoring: a need of HEls to monitor their performance and visibility in the local, national or regional environment;

2. Accountability: the growing importance of accountability to demonstrate to non-academic stakeholders (government, industry, wider public) the value of research and of the public investment in it;

3. Decision-making: the need to help decision-making, especially in case of resource allocation;

4. Understand: the new capacity to understand how research leads to impact thanks to data.

We propose to adapt this broad framework to specific needs of Higher Education Institutions: effective RIS can have positive impact on various levels of activity.

- On the level of external partners (quadruple-helix-actors) and integration in regional and national policy definition: the system can increase transparency of research activity, making the 
university more accessible from the outside, and encouraging partnerships. It can also serve for instance as a tool supporting regional Smart Specialisation Strategy and the related Entrepreneurial Discovery Process.

- On the level of top-management: evidence-based definition of strategy can in principle support an effective piloting of $\mathrm{HEI}-$ for reasons we will detail below, the direct use of quantitative indicators for decision making has been however put aside for the short term.

- On the level of mid-level management, units and individual researchers: if the system provides a sufficiently fine granularity and the capacity to explore output from the content side (which is the perspective of the researchers), this can lead to an increased capacity to meaningfully gauge one's competences and ways to communicate them.

- On the level of accountability: a performant RIS enables a stronger evidence-based communication generating more value for internal and external transparency and communication by thorough publicly visible analysis and reporting.

Beyond these benefits, a performant RIS may allow the university to actively contribute to the Open Science movement by building up potentially transferable expertise and infrastructure.

\section{MANAGING BIASES OF THE INDICATORS}

However, designing a pertinent Research Information System (RIS) requires a clear view of biases induced by existing quantitative measures of research production and quality.

Criticism against the measure of research impact via standardised quantitative indicators have accompanied their rise. As exemplary, we briefly relate the debate surrounding impact assessment via bibliometric indicators, notably citation counts.

Citation counts have become the reference in bibliometrics for impact assessment. Hicks and Merkels (2013, p. 4) recall the main areas of controversy: "Disagreement centers on what citations measure (quality would be ideal, but impact is more realisticl), distortions of citation counts (high rate achieved through negative citation, citation circles, self-citation etc.) or the value of contributions that do not appear in papers (database curation, creation of new materials or organisms, or increased human capital for example)". Other biases have been identified: cumulative effect in favour of advanced researchers (for the specific case of h-index - Gingras 2014, Penfield and al. 2014, Bornmann and Marx 2013); difficulties to measure long-term impact (Gingras 2014), thus discouraging risky upstream research whose impact may, if ever, be visible only after years (Wang et al. 2017); biases due to constitution of existing databases (overrepresentation of journals from United Kingdom and United States; underrepresentation of certain fields - SSH - or formats - monographs, conferences, etc.).

Therefore, we considered carefully the indicators that were to be included in the PSL Research Information System. We prioritized normalized indicators (FWCI by Scopus; proportion of publications in the Top 1\%/Top 10\%). We have chosen to complement bibliometric indicators with other types of metrics (e.g. ERC and Marie Curie grants and other types of European projects, which are a good proxy for measuring research quality at European research-intensive universities). We also added metrics which were not focused on impact (volume of internal and external collaborations). The system provides direct access to all corpuses of publications related to the quantitative indicators featured to meet the requirement of transparency and to enable in-depth analyses and criticism.

\section{MAIN USES IDENTIFIED}

The main uses that are currently implemented are:

- Monitoring: as a young university which still has to be confirmed by French government (cf French policy of "Idex" - mergers of best HEls in France - since 2011), PSL's ability to collect reliable and precise data on various subjects (academic and research staff by laboratories / fields; consolidated budgets for research, number of research projects, etc.) is key to enhance its own institutional credibility towards external stakeholders (International Idex jury, government). Moreover, the complexity of the Parisian landscape of Higher Education and Research (one lab may be affiliated simultaneously to PSL, Sorbonne Université and to the national research organism CNRS) makes it necessary to have precise data on the status of the several researchers within each lab.

- Accountability: added-value of the merger-process must be proven. Specific indicators were identified to illustrate synergies made possible by the merger (increase of co-publications between merged institutions, increase of national and regional research projects, etc.). More generally, strategic dialogue with French government relies partly on quantitative analyses; it is crucial for PSL to provide reliable data proving PSL's position as university of excellence in France. By now, we have focused on available indicators (increasing internal collaborations to demonstrate synergies, number of ERC grants per researchers, number of publications in the Top $1 \%$ / Top $10 \%$, etc.).

- Communication: quantitative indicators based on transparent data sources help PSL to position itself as a major Higher Education Institution in France (and even in Europe). Quantitative indicators, rather than reputation, objectify (or: seem to objectify) the scientific potential of PSL (and, then, the interest of the merger). Objective results (through two international rankings: THE and $\mathrm{OS}$, but also through consolidated bibliometric indicators) are likely to increase significantly researchers' and students' sense of belonging.

For the first stage of development and implementation, we focused on metrics and uses that were to increase PSL's strategic capacity by providing reliable and transparent data.

\section{DIFFICULTIES}

The difficulties to overcome are numerous:

- Some of the metrics are either themselves of doubtful quality, e.g. the biases introduced by bibliometric citation counts, so that their use in policy-definition is problematic or are too complicated to be sensibly used beyond a small circle of experts. 
- The grasp of central management on the activities of units and individual researchers is typically low, so that the formulation of strategy and the conscious development of the research portfolio is difficult. The interest which researchers have for such matters is equally low. However, when evidence-based strategy definition is used as a tool for collective strategic decision making, it can support strategic thinking throughout the organisation. This is especially efficient when the whole process is fully transparent and co-designed with all actors at different levels: e.g. unit directors, research programs, schools. Building tools not only for top management, but that researchers can use themselves strengthens the engagement of communities towards the creation of a comprehensive RIS.

- Quality granular data on science and innovation activities and results is typically lacking, so that data must first be painstakingly acquired, and results can often only be published much further down the line (3 years of data gathering without any output visible to the community are not rare - enough to make a project lose impetus).

- A partial and segmented view of the research and innovation process, the value chains and of the overlapping institutional and public policies, due to a lack of integrated or interoperable data.

- A difficulty to define pertinent priority areas because of the difficulty to classify R\&D activities and results beyond journal taxonomies provided by bibliometric data providers (try to grasp "Cryptography" or "Breast Cancer" via those taxonomies, especially since the Scopus taxonomy is established on the level of the journals and not on the level of the individual publication).

- A gap between experts and stakeholders, between users and providers of data and analysis, due to different vocabularies, knowledge and experiences.

- Concretely in our case: PSL is a complex entity: it is a university system on the path of becoming a single university. This means, that heterogenous institutions with varying levels of data quality and availability are collaborating in this project accompanied by political unrest during a time of deep change in the French and especially Parisian HERI landscape. Related difficulties include:

- lack of pressure from the relevant ministries;

- lack of interest of the individual researchers whose engagement is yet essential at least for data curation and quality management purposes;

- lack of infrastructures at PSL member institutions;

- the complex and intricate Parisian HERI system with many research units being shared among more than one actor entailing the need for collaboration.

- In France, no strong evaluation procedures are in place that could put pressure on institutions to adopt a data policy as it happened in the UK and in Italy.

\section{METHODOLOGY}

We are sceptical towards pharaonic data projects at the national level, of which we have seen a few and which for now have not led to the breakthroughs they promised. ${ }^{6}$ We do not wish to imply that such projects are nonsensical per se. We simply wish to say, that institutions should not wait for such projects to move forward. Institutions must take up the initiative themselves whilst ensuring that their actions can be adapted a posteriori to other initiatives or overarching standardisation efforts.

We adopted a methodology based on the presumption that engaging the community and keeping it engaged are key, if we want the system to be adopted and useful. We therefore adopted two principles:

- Do not place more work on people for data gathering than you absolutely must, because increasing the workload of people without providing quick return endangers engagement.

- Provide tangible returns pertinent for the stakeholders as early as possible in the process to establish and maintain legitimacy of the project. Define most needed uses as a first step. Target uses that can be profitable also for faculty staff (not only for the top management).

Building on these principles, instead of starting to build a comprehensive data warehouse, we started with a single dashboard as a pilot based principally on open data and bibliometric data to show the community what the capacities of the RIS might be with minimal supplementary workload on the community.

From this initial exercise, we go on to the definition of specific fields of interest and defined by focus groups and discussed with a wider audience during workshops and integrate further data (open if available or internal if not) to provide further pertinent indicators or to increase data quality by integrating internal data. However, the idea is to develop the RIS step by step:

$\rightarrow$ Definition of an indicator $\rightarrow$ integration of data $\rightarrow$ rendition of results to stakeholders $\rightarrow$ restart.

\section{TECHNOLOGY}

From the technological point of view, the requirements were the following:

- The system should be a lightweight and minimally invasive data federation and integration tool, that can be plugged into existing sources. It should not require the adoption of a specific new data curation system by each of the original source curators.

- The system should link internal data with external and ideally open data sources, since this achieves 2 goals:

- it is a highly effective means for quality assessment since it allows us to compare the data we received internally to external quality sources. E.g. we compare internal information on European projects to the EU's own CORDIS database.

- we can reach a high level of synergy by using available information from different sources.

This way, we can ensure the quality of the data whilst enriching the external sources with detailed information only available from local providers. This approach allowed us for instance to track down the lab level participation in European projects (CORDIS only provides institutional level information) by combining the EU's base CORDIS ${ }^{7}$ with information from the Open Data service ScanR ${ }^{8}$ operated by the French Ministry of Higher Education. 
- It should rely on open standards, be open source and under active development by a reliable community, because this ensures the independence of the client from any service provider (including SIRIS $^{9}$ ). It also should be implemented among a relevant range of actors to ensure its sustainability.

The system is based on Semantic Web technologies, using OntologyBased Data Access and of Linked Data approaches. This is a state-ofthe-art framework which enables it to federate heterogeneous sources under a common vocabulary (ontology) without reforming the data curation at the local level.

The system conforms to the standards of the World Wide Web Consortium (WC3) ${ }^{10}$ and to the Europe 2020 Strategy of the European Union ${ }^{11}$ that advocates and promotes the use of Linked Open Data and Semantic Web technologies. ${ }^{12}$ It includes an endpoint in the standard SPAROL language, which will allow the user to participate in Linked and Open Data initiatives in the future in order to increase visibility. The ontology is entirely compatible with VIV0-ISF ${ }^{13}$ originally developed at Cornell University and with its European counterpart, the Common European Research Information Format (CERIF) ${ }^{14}$. This is essential for the future development and for the independence of the system from specific providers and to maintain sovereignty over its data at all times. VIVO-based systems are already implemented by over 140 academic actors ${ }^{15}$ across 25 countries from Cornell and the MIT to UCLA, the presumption of durability is therefore warranted. Institutions that have implemented Linked Open Data approaches include National Statistical Agencies like the French INSEE ${ }^{16}$ or the Italian ISTAT ${ }^{17}$, as well as publishers, most notably Nature (Springer $)^{18}$.

\section{RESULTS}

The current dashboard provides numerous elements for strategic analysis. The perimeters of analysis can be freely defined on the level of the research units (of which PSL has roughly 180), so that any combination of units can be aggregated for analysis. Available indicators include

- Bibliometric indicators for around 300 categories weighted against France, Europe or the world including benchmark modules with other French and European universities $\rightarrow$ useful to gauge strengths and weaknesses in the overall profile.

- EU projects: participation and funding filterable by programme weighted against France or Europe $\rightarrow$ top-level performance on the international scene

- Internal networks between units: co-publications and internal projects $\rightarrow$ useful to track real collaborations (and their respective intensity) in all the fields.

- External networks: Co-publications and EU projects $Đ$ evaluate partnerships
More importantly, however, we have had numerous meetings with internal and external stakeholders and were able to assess more in detail the requirements and expectations. The transition from a first quickly done, largely top-down phase to a slower pace led by participatory design principles sees the project now on presumably much firmer position.

The added-value of this project until now consists in:

- Providing University's top management with consolidated data (staff, number of publications and impact, number of projects, range of internal collaborations, etc.), thus enhancing its credibility as an organisation able to define a strategy (both towards internal - the schools composing the University - and external stakeholders)

- Providing reliable data that may support lobbying and communication strategy

- Favouring a cultural change towards data use, also within academic community

- Supporting the development of other data-based tools for alternative uses (showcase PSL's areas of scientific expertise, build scientific maps on general or specialized topics, etc.)

- Helping to better define the way bibliometrics could be implemented to support decision-making.

\section{REFERENCES}

Bornmann, L. and Marx, W. (2013). How good is research really? EMBO Reports, 14, 226-230.

Calvanese, D., De Giacomo, G., Lembo, D., Lenzerini, M., Poggi, A., Rodríguez-Muro, M. and Rosati. R. (2009). Ontologies and databases: The DL-Lite approach. In Tessaris, S., Franconi, E. (Eds.), Reasoning Web. Semantic Technologies for Information Systems - 5th International Summer School Tutorial Lectures RW Lecture Notes in Computer Science, vol. 5689 (pp. 255-356). Cham: Springer.

Calvanese, D., De Giacomo, G., Lembo, D., Lenzerini, Rosati. R. and Ruzzi. M. (2009). Using OWL in data integration. In: Semantic Web Information Management - A Model-Based Perspective (pp. 397-424). Cham: Springer.

Deem, R., and Brehony, K. J. (2005). Management as ideology: The case of 'new managerialism' in higher education. Oxford review of education, 31, 217-235.

Dunleavy, P., Margetts, H., Bastow, S., and Tinkler, J. (2006). New public management is dead-long live digital-era governance. Journal of public administration research and theory, 16, 467- 494. 
Ferlie, E., Musselin, C. and Andresani, G. (2008). The steering of higher education systems: A public management perspective. Higher education, 56, 325-348.

Foray, D. and Goenaga, X. (2013). The goals of smart specialisation. European Commission, Joint Research Centre, S3 Policy Brief Series No. 1.

Gianelle, C. and Kleibrink, A. (2015). Monitoring Mechanisms for Smart Specialisation Strategies. European Commission, Joint Research Centre, S3 Policy Brief Series No. 13.

Gingras Y. (2014). Les dérives de l'évaluation de la recherche: du bon usage de la bibliométrie. Paris: Raisons d'agir.

Haustein, S. and Larivière, V. (2015). The use of bibliometrics for assessing research: Possibilities, limitations and adverse effects. In Welpe, I.M., Wollersheim, J., Ringelhan, S. and Osterloh, M. (Eds), Incentives and performance (pp. 121-139). Cham: Springer.

Hicks, D. and Melkers, J. (2013). Bibliometrics as a tool for research evaluation, in Link, A.N. and Vonortas, N.S. (Eds), Handbook on the Theory and Practice of Program Evaluation, (pp. 323-349). Cheltenham: Edward Elgar Publishing.

Hicks, D., Wouters, P., Waltman, L., de Rijcke, S., and Rafols, I. (2015). Bibliometrics: the Leiden Manifesto for research metrics.

Mosca, A., Rondelli, B. and Rull. G. (2017). The OBDA-Based "Observatory of Research and Innovation" of the Tuscany Region. Proceedings of the Joint Ontology Workshops 2017 Episode 3: The Tyrolean Autumn of Ontology, Bozen-Bolzano.

Mosca, A., Roda, F. and Rull, G. UNiCS - The ontology for Research and Innovation policy making. Submitted.

Penfield, T., Baker, M. J., Scoble, R. and Wykes, M. C. (2014). Assessment, evaluations, and definitions of research impact: A review. Research Evaluation, 23, 21-32.

Radosevic, S. (2017). Assessing EU Smart Specialization Policy in a Comparative Perspective. In: Radosevic, S., Curaj, A., Gheorghiu, R., Andreescu, L., Wade, I. (Eds) Advances in the Theory and Practice of Smart Specialization. London: Academic Press.

Thoenig, J. and Paradeise, C. (2016). Strategic Capacity and Organisational Capabilities: A Challenge for Universities. Minerva. 54, 293-324.

Thoenig, J. and Paradeise, C. (2015). In Search of Academic Quality, Palgrave Macmillan.

Wang, J., Veugelers, R. and Stephan, P. (2017). Bias against novelty in science: A cautionary tale for users of bibliometric indicators. Research Policy, 46, 1416-1436.

\section{AUTHORS}

\section{EMMANUEL BASSET}

Director of Research \& Graduate Education, PSL University Paris,

60 rue Mazarine, Paris, 75006 (France)

E: emmanuel.basset@psl.eu

\section{MATTHIAS HEUSER}

Strategy Consultant at SIRIS Academic SL

Av. Francesc Cambó 17, Barcelona, 08003 (Spain)

E: matthias.heuser@sirisacademic.com

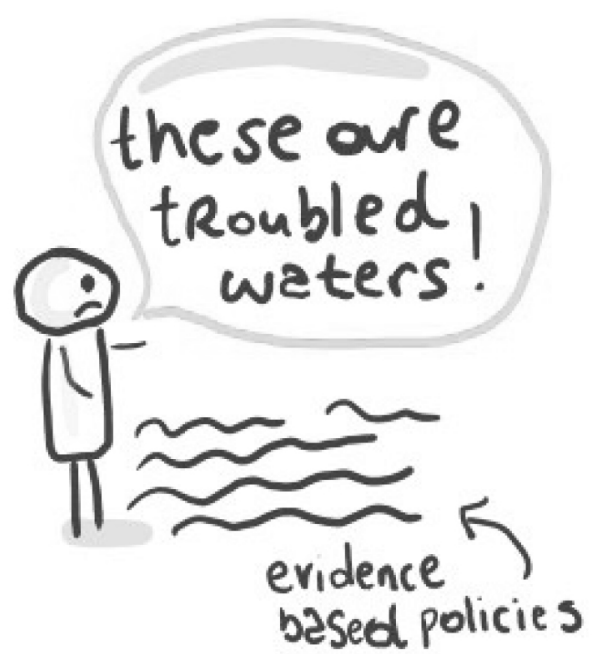

